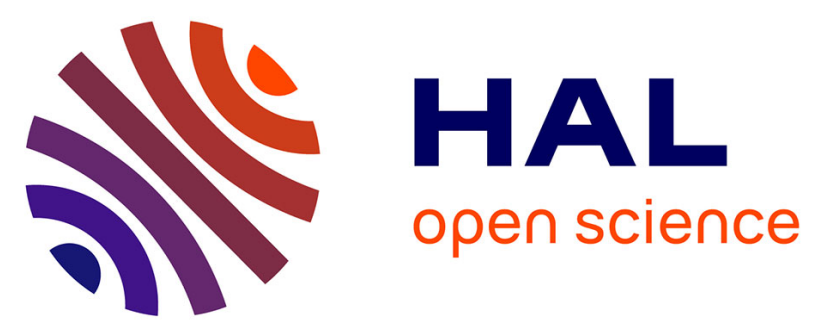

\title{
Emergence of drug tolerance in cancer cell populations: an evolutionary outcome of selection, non-genetic instability and stress-induced adaptation
}

Rebecca H. Chisholm, Tommaso Lorenzi, Alexander Lorz, Annette K. Larsen, Luís Almeida, Alexandre Escargueil, Jean Clairambault

\section{To cite this version:}

Rebecca H. Chisholm, Tommaso Lorenzi, Alexander Lorz, Annette K. Larsen, Luís Almeida, et al.. Emergence of drug tolerance in cancer cell populations: an evolutionary outcome of selection, nongenetic instability and stress-induced adaptation. 2015. hal-01111271

\author{
HAL Id: hal-01111271 \\ https://hal.science/hal-01111271
}

Preprint submitted on 30 Jan 2015

HAL is a multi-disciplinary open access archive for the deposit and dissemination of scientific research documents, whether they are published or not. The documents may come from teaching and research institutions in France or abroad, or from public or private research centers.
L'archive ouverte pluridisciplinaire HAL, est destinée au dépôt et à la diffusion de documents scientifiques de niveau recherche, publiés ou non, émanant des établissements d'enseignement et de recherche français ou étrangers, des laboratoires publics ou privés. 


\section{Emergence of drug tolerance in cancer cell populations: an evolutionary outcome of selection, non-genetic instability and stress-induced adaptation}

Running title: Modeling reversible drug tolerance in cancer cell populations

Keywords: Cancer modelling; reversible drug tolerance; phenotype evolution; non-genetic instability; stress-induced adaptation

Authors: Rebecca H. Chisholm ${ }^{1,2,3, *}$, Tommaso Lorenzi ${ }^{1,2,3}$, Luis Almeida ${ }^{1,2,3}$, Annette K. Larsen ${ }^{4,5}$, Alexander Lorz ${ }^{1,2,3}$, Alexandre Escargueil ${ }^{4,5}$, Jean Clairambault ${ }^{1,2,3}$

Author's Affiliations: ${ }^{1}$ INRIA-Paris-Rocquencourt, MAMBA Team, Domaine de Voluceau, BP105, 78153 Le Chesnay Cedex, France; ${ }^{2}$ Sorbonne Universités, UPMC Univ Paris 06, UMR 7598, Laboratoire Jacques-Louis Lions, F-75005, Paris, France; ${ }^{3}$

CNRS, UMR 7598, Laboratoire Jacques-Louis Lions, F-75005, Paris, France; ${ }^{4}$ Sorbonne Universités, UPMC Univ Paris 06, F-75005, Paris, France; and ${ }^{5}$ INSERM, UMR_S 938, Laboratory of "Cancer Biology and Therapeutics", F-75012, Paris, France.

Corresponding Author: Rebecca Chisholm, E-mail: rebecca.chisholm@inria.fr, INRIA-Paris-Rocquencourt, MAMBA Team, Domaine de Voluceau, BP105, 78153 Le Chesnay Cedex, France, Phone: (+33) 144278516

Financial Support: The research leading to this paper was (partially) funded by the french "ANR blanche" project Kibord: ANR-13-BS01-0004. TL was supported by the Fondation Sciences Mathématiques de Paris (FSMP) and by a public grant overseen by the French National Research Agency (ANR) as part of the "Investissements d'Avenir" program (reference: ANR-10-LABX-0098). 
Disclosure of Potential Conflicts of Interest: The authors disclose no potential conflicts of interest.

Word Count: Abstract: 342, Quick guide to equations and assumptions: 685, Body: 4927.

\section{Number of Figures: 7.}

\section{Abstract}

In recent experiments on isogenetic cancer cell lines, it was observed that exposure to high doses of anti-cancer drugs can induce the emergence of a subpopulation of weaklyproliferative and drug-tolerant cells, that display markers associated with cancer stem cells. After a period of time, some of the surviving cells were observed to change their phenotype to resume normal proliferation, and eventually repopulate the sample. Furthermore, the drug-tolerant cells could be drug resensitized following drug washout. Here we propose a theoretical mechanism for the transient emergence of such drug tolerance. In this framework, we formulate an individual-based model and an integro-differential equation model of reversible phenotypic evolution in a cell population exposed to cytotoxic drugs. The outcomes of both models suggest that selection, non-genetic instability, stress-induced adaptation and the interplay between these mechanisms can push an actively proliferating cell population to transition into a weakly-proliferative and drugtolerant state. Hence, the cell population experiences much less stress in the presence of the drugs and, in the long run, reacquires a proliferative phenotype, due to selection pressure and phenotypic fluctuations. These mechanisms can also reverse epigenetic drug tolerance following drug washout. Our study highlights how the transient appearance of the weakly-proliferative and drug-tolerant cells is related to the use of high-dose therapy. 
Furthermore, we show how stem-like characteristics can act to stabilize the transient, weakly-proliferative and drug-tolerant subpopulation for a longer time window. Finally, using our models as in silico laboratories, we propose new testable hypotheses that could help uncover general principles underlying the emergence of cancer drug tolerance.

\section{Major findings}

The application of our mathematical models to a recently studied experimental system (where there is evidence of an epigenetically regulated and transient drug-tolerant phenotype in an isogenetic cancer cell line), suggests that mechanisms based on fundamental laws of biology, namely a combination of selection, non-genetic instability mediated by phenotypic noise and stress-induced adaptation, can (reversibly) push an activelyproliferating and drug-sensitive cell population to transition into a weakly-proliferative and drug-tolerant state, which will eventually facilitate the emergence of more potent, proliferating and drug-tolerant cells.

\section{Introduction}

The interplay between intra-tumor heterogeneity $[1,2]$ and perturbations in the tumor micro-environment [3-6] plays a key role in the emergence of drug resistance in cancer cell populations. Intra-tumor heterogeneity results mainly from genetic modifications (e.g., mutations) leading, through a Darwinian-like process, to the selection of tumor cells expressing phenotypes adapted to the local environment $[2,7]$. However, intra-tumor heterogeneity can also emerge from non-genetic processes mediated by either stochastic events or epigenetic modifications [8-10]. In fact, perturbations of the tumor micro-environment

induced by cytotoxic agents may 'instruct' cells to enter a more stress-resistant phenotype, 
which can be passed on to subsequent cell generations [11].

In recent experiments, performed on a genetically homogeneous population of lung (PC9) cancer cells, Sharma and co-workers [12] showed that epigenetically regulated changes in phenotype can play an important role in the development of reversible drug tolerance. During these experiments, a small subpopulation of drug-tolerant cells was consistently detected, that could maintain viability in the presence of high-dose drug therapy. These Drug-Tolerant Persisters (DTPs) were shown to be non-proliferative and display markers specific to Cancer Stem Cells (CSCs). After a period of time, approximately 20 percent of DTPs changed their phenotype to resume normal proliferation and lost CSC markers, still in the constant presence of the drugs. The resulting cells were labelled the Drug-Tolerant Expanded Persisters (DTEPs) (Fig. 1(a)). We may infer from these observations that two phenotypically distinct cell sub-clones subsequently emerge: a first one with a survival-prone but non-proliferative phenotype (the DTPs), and a second one, able to survive in extremely hostile conditions and proliferate (the DTEPs). Interestingly, both DTPs and DTEPs could be drug resensitized by drug-free passaging.

Therefore, the three distinct subpopulations-PC9s, DTPs and DTEPs - that compose the whole PC9 population at various times during drug treatment, although genetically identical, possess different functional phenotypes. Most notably, they can be characterized by their respective levels of survival potential in extreme environments and proliferation potential, which are two traits that have important clinical implications for cancer patients [13]. But what is driving the evolution of phenotype observed in the PC9 cancer cells?

During drug therapy, the average level of survival potential (i.e., the average level of robustness towards life-threatening events in extreme conditions) in the PC9 cell population is non-decreasing over time. We argue that this can be explained by the interaction 
between two mechanisms. The first mechanism is non-genetic instability mediated by phenotypic noise [14]. The term phenotypic noise, also known as biological noise [9, 10], refers to the fluctuations in phenotype due to processes which may be intrinsic or extrinsic to the cell [15], and may include, but are not limited to, noise related to gene expression [16,17], protein production [18] and DNA methylation. Fluctuations in phenotype give rise to phenotype heterogeneity and can result in the transition of drug-sensitive cells into a robust phenotype $[4,10,19]$. These cells are able to survive drug exposure, and hence the second mechanism, selection, can cause the most robust cells to become the majority in the population.

The dynamics of the average level of proliferation in the cell population is even more interesting. Initially, as the majority phenotype transitions from the PC9 phenotype to the DTP phenotype, the average proliferation level drops. However, after a period of time, as DTEPs emerge from DTPs, the average level of proliferation increases back to normal. The interplay between non-genetic instability and selection could explain the emergence of the DTEPs from the DTPs. Nevertheless, these mechanisms alone cannot account for the initial drop in the average level of proliferation of the surviving cell population. More specifically, if we assume there are no DTPs present in the initial PC9 population, then we would expect (small and rare) fluctuations in phenotype, combined with selection, to result in the emergence of DTEPs directly from PC9s (so that proliferation levels remain high). The transient dominance of DTPs indicates that another process is involved.

A natural explanation is that there are a small number of DTPs present in the initial population of PC9 cells. These cells survive the initial drug therapy, and are subsequently selected for in the presence of the drugs. However, this scenario is only plausible if there are some DTPs present from the beginning. If this is not the case, then we propose that the DTP phenotype must be stress-induced $[11,20,21]$. Recent work on colorec- 
tal cancer cells by Kreso and co-workers [20] showed that, in response to chemotherapy, actively proliferating cells were preferentially eliminated compared with other relatively dormant progeny. The authors suggest that these cancer cells may use dormancy (i.e., mere survival without differentiation, senescence nor commitment into the division cycle) as an adaptive strategy. Accordingly, we suggest that, if there are no DTPs present in the initial PC9 population, then stress induces the PC9 cells to adopt a more DTP-like phenotype $[11,20]$. In this adaptive scenario, PC9 cells are induced to redistribute their finite energy resources away from proliferation-oriented tasks and towards survival-oriented tasks (for example, nucleotide metabolism, DNA synthesis and repair [22]). On the other hand, robust cells, with a high survival potential, experience much less stress in the presence of the drugs. They have no need to redistribute their energy resources away from proliferation-oriented tasks. Accordingly, in response to drug exposure, one will expect a population of mostly drug-sensitive PC9 cells to experience an initial sharp drop in average proliferation levels. Only when levels of resistance towards drug damage increase sufficiently, due to fluctuations in phenotype and selection, will cells be able to reallocate resources back into proliferation-oriented tasks, and resume normal proliferation [23, 24].

Motivated by these considerations, here we propose an Individual-Based (I-B) computational model [11,25-27] and an Integro-Differential Equation (IDE) model [28-30] of the phenotype evolution observed in the cell lines of Sharma and co-workers [12]. Such models can be used as in-silico laboratories to highlight stylized facts, and uncover mechanisms that underlie emergent features of cancer cell populations. The I-B computational model allows an intuitive and flexible description of the system at hand, while the IDE model makes it possible to study the system in terms of qualitative and asymptotic analysis, and is computationally less expensive.

In particular, we focus on the evolution of two phenotypic traits which show substantial 
variability during drug treatment and after drug washout in the PC9 cell lines: a cell's survival potential and proliferation potential [13]. These models rely on the assumption that PC9 cells possess significant phenotypic plasticity. Furthermore, we assume that, during drug treatment, the surviving cell population can consist of cells residing within a spectrum of intermediate phenotypic states, ranging from PC9 through to DTP, and to DTEP. This idea is consistent with observations in other genetically identical cancer cell populations, where there exists significant functional phenotype heterogeneity, even in homogeneous environments $[4,11,12,20]$. For example, although epithelial-mesenchymal transition is often portrayed as a switch between two distinct states, in fact it has been shown that epithelial cells progressively acquire mesenchymal features at the same time as they lose epithelial attributes [31]. In the same way, during drug treatment, we assume that the PC9s can gradually acquire drug tolerance at the same time as they gradually change their proliferation ability.

In the presence of high-dose drug therapy, we illustrate the ability of the models to recover the evolution of the PC9 cells into DTPs and finally into DTEPs, and also the drug resensitization of DTPs and DTEPs. Showing this, we can then argue that the models have validity for suggesting plausible evolutionary mechanisms underlying drug tolerance. Therefore, we utilize our I-B computational and IDE models in parallel to address the following questions:

Q1. Is non-genetic instability necessary in the development of drug tolerance?

Q2. How do the evolutionary dynamics of the cell population compare between the regimes of low and high drug dose?

Q3. Is it possible to determine whether therapy induces the DTP phenotype?

Q4. What is the role of stem-like characteristics of the DTP cells in the development of drug 
tolerance?

Our study highlights the important role of non-genetic instability in the emergence of DTEPs, and also leads us to propose experiments which may help to assess whether there are DTPs present ab initio, and whether PC9 cells are undergoing stress-induced adaptation of their phenotype. Finally, our models predict that the transient dominance of the DTP subpopulation is regulated by their stem-like characteristics and is a direct result of the high doses of chemotherapeutic drugs used in [12].

\section{Quick guide to equations and assumptions}

We describe the evolution of phenotype in a well-mixed PC9 cancer cell population exposed to cytotoxic drugs (whose concentration at time $t$ is $c(t)$ ), using both an I-B and an IDE formalism. We consider only the case of constant infusion of cytotoxic drugs (i.e., $c(\cdot):=c>0)$ and characterize the state of each PC9 cell by its level of expression of two phenotypic traits: survival potential and proliferation potential. In this framework, we identify a PC9 cell as having a low value of survival potential and a high value of proliferation potential, a DTP cell as having a high value of survival potential and a low value of proliferation potential, and a DTEP cell as having a high value of survival and proliferation potential (schematized as in Fig. 1(b)). We model the three key biological mechanisms of phenotype evolution in the PC9 cancer cells, namely selection, stress-induced adaptation and non-genetic phenotype instability, by using three separate mathematical strategies, which are tailored to fit the I-B and the IDE formalisms. Specifically, we model natural selection through a proliferation probability $p$ and a death probability $d$, which depend on the levels of proliferative and survival potentials of the cells, as well as on the cell micro-environment at time $t$. Stress-induced adaptation of cell proliferation level, which occurs in the presence of the drugs, and depends on the level of survival potential, is 
modeled as an advection term. It leads to a decrease in the level of proliferation, and varies with the survival potential of the cell and the concentration of the drugs. Finally, non-genetic phenotype instability is modeled by stochastic fluctuations, or diffusion, in the levels of cell proliferation and survival potentials.

\section{Individual-based model}

In the I-B formalism, we consider each cell as an individual agent, and label it by an index $0 \leq i \leq N(t)$, where $N(t) \geq 0$ is the size of the population at time $t \in[0, T]$, and $T$ is the end time of the simulation. The normalized expression levels of the survival-potential and proliferation-potential traits, in each cell $i$ at time $t$, are modeled, respectively, by the random variables $X_{i}(t):[0, \infty) \rightarrow[0,1]$ and $Y_{i}(t):[0, \infty) \rightarrow[0,1]$. We simulate the evolution of the cell population in discrete time, according to the algorithm pictured in Supplementary Fig. S2. Over the time interval between two successive time instants $t$ and $t+\Delta t$, we first allow each cell $i$ either to proliferate, undergo apoptosis or remain in a quiescent state according to the respective probabilities $p\left(X_{i}, Y_{i}, N(t)\right) \Delta t, d\left(X_{i}, c(t)\right) \Delta t$ and $1-\Delta t\left(p\left(X_{i}, Y_{i}, N(t)\right)+d\left(X_{i}, c(t)\right)\right)$. If a cell proliferates, we assume both daughter cells inherit the parent's trait values. This is consistent with experimental observations in cancer cell lines, which suggest that a cell's "epigenetic memory" can last multiple cell generations [18]. After all cells have undergone one iteration of the proliferation and death process, and returned to the quiescent state, we then let each cell update its trait values according to the following system of discretized Stochastic Differential Equations:

$$
X_{i}(t+\Delta t)=X_{i}(t)+\underbrace{D \sqrt{\Delta t} W_{i}^{1}(t),}_{\begin{array}{c}
\text { non-genetic } \\
\text { instability }
\end{array}} Y_{i}(t+\Delta t)=Y_{i}(t)+\underbrace{D \sqrt{\Delta t} W_{i}^{2}(t)}_{\begin{array}{c}
\text { non-genetic } \\
\text { instability }
\end{array}}+\underbrace{\Delta t}_{\begin{array}{c}
\text { stress-induced adaptation } \\
\text { of the proliferation level }
\end{array}}
$$


where $\bar{v}$ represents the average sensitivity of the cell proliferative potential to stressinducing agents, and $D$ is the average rate of phenotypic fluctuations.

\section{Integro-differential equation model}

In the IDE formalism, we consider the cell population to be structured by two continuous, real variables $x \in[0,1]$ and $y \in[0,1]$, which represent, respectively, the normalized expression levels of the survival-potential and proliferation-potential traits. The population density of cancer cells is modeled by the function $n(x, y, t) \geq 0$, where the global population density at time $t \in[0, T]$ is computed as $\varrho(t)=\int_{0}^{1} \int_{0}^{1} n(x, y, t) \mathrm{d} x \mathrm{~d} y$, and the time evolution of $n$ is governed by the following equation:

$$
\frac{\partial n}{\partial t}(x, y, t)+\underbrace{\frac{\partial}{\partial y}(v(x, c(t) ; \bar{v}) n(x, y, t))}_{\begin{array}{c}
\text { stress-induced adaptation } \\
\text { of the proliferation level }
\end{array}}=\underbrace{[p(x, y, \varrho(t))-d(x, c(t))] n(x, y, t)}_{\text {selection }}+\underbrace{\beta \Delta n(x, y, t),}_{\begin{array}{c}
\text { non-genetic } \\
\text { instability }
\end{array}}
$$

where $\beta$ is related to the parameter $D$ from the I-B model through the relation $\beta=D^{2} / 2$.

\section{Extended individual-based model}

In the extended I-B model, we keep the birth and death process unchanged from the original I-B model. However, instead of allowing the DTPs to update at each time step, we consider them to be stem-like so that they do not change their phenotype, unless they proliferate. When a DTP undergoes proliferation, we allow its offspring to update according to $p_{1}, p_{2}$ and $p_{3}$ which represent, respectively, the probabilities for symmetric self-renewal, asymmetric self-renewal and symmetric differentiation. The update mechanisms of the PC9s and DTEPs remain unchanged. This new algorithm is pictured in Supplementary Fig. S3. 
The assumptions on the functions $p, d$ and $v$ are summarized in Supplementary Tables S1-S2. All definitions of the parameter functions are provided in the Supplementary Material.

\section{Results}

For each scenario presented below, we consider the concentration of cytotoxic drugs (i.e., the parameter c) to be high if it is greater than, or equal to, the $\mathrm{LC}_{90}$ dose (defined as the drug dose required to kill $90 \%$ of the total cell population in the initial stage of drug therapy, before the population starts to recover). On the other hand, we assume that a drug dose is low if it is smaller than, or equal to, the $\mathrm{LC}_{50}$ dose (defined as the drug dose required to kill $50 \%$ of the total cell population in the initial stage of drug therapy, before the population starts to recover). The end-time of the simulations is represented by the parameter $T$, and is defined as the time required for the global population density to reach equilibrium.

\section{Mathematical models recover the population level dynamics of the PC9 cells}

To determine whether our models can reproduce the experimental observations of the PC9 cancer cell lines during drug therapy, as reported in [12], we numerically solve the IDE model and simulate the I-B model for a high dose of the cytotoxic drugs. Both models can successfully reproduce the important population level dynamics of the PC9 cells, namely the extinction of the PC9 cells, followed by the transient appearance of DTPs and the emergence of the DTEP subpopulation.

In Fig. 2, we present numerical solutions from the I-B and IDE models for the case 
when there are a few DTPs present in the initial population (i.e., $5 \%$ of the global population density), and when there is no stress-induced adaptation of the proliferation potential. Fig. 2(a) displays the trajectories of the mean phenotypic expression of the population. It is clear that, as time progresses, the mean phenotypic expression changes from being PC9-like (high proliferation and low survival potentials), to being DTP-like (low proliferation and high survival potentials) and finally to being DTEP-like (high proliferation and survival potentials). Such dynamics are also shown in Fig. 2(c)-(j), which displays the phenotypic distribution at different times during drug therapy. In Fig. 2(b), the plot of the global population density as a function of time reveals that, shortly after drug exposure, there is a large reduction in cell number (more than 90\%), followed by a time of relatively constant population level, before the population recovers back to normal levels. Similar results can be obtained when there are no DTPs present in the initial population, and when we include stress-induced adaptation of proliferation levels (we present numerical solutions for these cases, from the IDE model in Fig. 4(a)-(b) and the top rows of Figures S5-S6).

Fig. 2 also shows that we can obtain a good match for the dynamics predicted by the I-B model with that predicted by the IDE model. We also achieve the same qualitative match for the other cases considered in this section. Therefore, with the exception of our study on the stem-cell characteristics of DTPs (where we use the extended I-B model), from now on we present numerical results obtained from the IDE model only.

In the experiments reported in [12], both the resistant DTPs and DTEPs can be drug resensitized by drug-free passaging. To test whether this is also the case in our models, we numerically solve the IDE model and simulate the I-B model for a period of time with high doses of cytotoxic drugs, so that either DTPs or DTEPs are left as the dominant subpopulation. We subsequently switch the drug concentration off to simulate 
drug washout, and allow the system to continue to evolve. We find that the models can successfully reproduce drug resensitization of the DTPs and DTEPs (see Fig. 3). This result also holds after drug washout following low-dose drug therapy (see Supplementary Fig. S4).

\section{A1. Non-genetic instability is crucial for the emergence of DTEPs}

To investigate the role of non-genetic instability in the evolution of drug tolerance in PC9 cells, we analyse the phenotype evolution in the PC9 population, for different values of the average rate of phenotypic fluctuations (i.e., the parameter $\beta$ ) for a high dose of cytotoxic drugs. We consider two hypothetical scenarios:

Scenario (i) when the initial population consists of only PC9s and stress-induced adaptation of the proliferation level is present;

Scenario (ii) when a small number of DTPs are present initially and stress-induced adaptation of the proliferation level is absent.

For the first scenario, our model predicts that if the average rate of phenotypic fluctuations is too large, then the PC9s will evolve directly into the DTEPs. Furthermore, if this rate is too small, then the PC9s will become extinct before they are able to express the more robust DTP phenotype. These predictions are illustrated in Fig. 4(a)-(b), where we plot the trajectories of the mean phenotypic expression of the population and the corresponding total cell density as a function of time, for three values of $\beta$ (see also Supplementary Fig. S5(a)-(b)).

For the second scenario, the model predicts that in the absence of non-genetic instability, the DTEPs cannot emerge from the DTPs. Therefore the DTP phenotype is stabilized inside the surviving population. Consequently, compared with the case when non-genetic 
instability is present, the long-term total global population density can be much smaller. In fact, if the concentration of the drugs is high enough, then the population can go extinct when non-genetic instability is absent. These predictions are illustrated in Fig. 4(c)-(d) (see also Supplementary Fig. S5(c)-(d)).

Therefore, we hypothesize that non-genetic phenotype instability is essential for the emergence of DTEPs, and for the development of drug tolerance in PC9 cells. Furthermore, the absence of non-genetic instability can result in the stabilisation of the DTP phenotype in the surviving population, and even extinction of PC9 cells during high-dose drug therapy.

\section{A2. A high dose of cytotoxic drugs is necessary for the transient dominance of DTPs}

We use our IDE model to investigate the evolutionary dynamics of the PC9 cells in a low drug-dose regime. For Scenario (i), described above, our model predicts that, ceteris paribus, decreasing the drug concentration can prevent the emergence of DTPs and speed up population recovery. These dynamics are illustrated in Fig. 5(a)-(b), where we plot the trajectories of the mean phenotypic expression of the population and the corresponding global population density as a function of time, for both a high dose and a low dose of the cytotoxic drugs. This is also true for Scenario (ii) (see Fig. 5(c)-(d)). Therefore, we can conclude that a higher dose of the cytotoxic drugs is a key ingredient to see the transient dominance of DTPs.

A complete set of comparisons between the high and low drug-dose regimes, for different values of $\bar{v}$ (the average sensitivity of the cell proliferative potential to stress-inducing

agents) and $\beta$ (the average rate of phenotypic fluctuations), are provided in Figures S5-S6 and support this conclusion. 


\section{A3. Low cytotoxic drug-dose experiments could establish whether the DTP pheno- type is stress-induced}

For the case when there are a few DTPs present in the initial PC9 population, our model predicts that in a low drug-dose regime, the emergence of a meta-stable subpopulation of DTP cells can require the presence of stress-induced adaptation, non-genetic instability in phenotype, and the proper interplay between them. This is illustrated in Fig. 6(b) where, for a given value of $\beta$ (the average rate of phenotypic fluctuations) the trajectories of the population mean trait levels pass through the region corresponding to the DTP phenotype, only when stress-induced adaptation is present. In fact, in order to allow for the emergence of a metastable subpopulation of DTP cells, a sufficiently high sensitivity of the cell proliferation potential to stress-inducing agents (i.e., the parameter $\bar{v}$ ) and a suitable value of $\beta$ are required. This is in contrast to the high drug-dose regime where DTPs emerge in the absence of stress-induced adaptation, and the evolutionary dynamics of the population are unaltered by changes in $\bar{v}$ (see Supplementary Fig. S6(c)-(d)).

Another interesting prediction from our model in the low-dose regime is that, if there are a few DTPs present in the initial PC9 population, and stress-induced adaptation is absent, then it is possible to see an increase in the average proliferative potential in the surviving population, followed by a decrease before a second increase (see Fig. 6). This is not predicted in the high-dose regime, nor is it predicted if there are no DTPs present initially, and it is related to the timing of the emergence of the DTEPs, and the disappearance of the PC9s.

Therefore, one way to determine whether there are pre-existing DTPs within the initial PC9 population, and whether stress-induced adaptation is occurring, would be to repeat the experiments of [12] with a low dose of the cytotoxic drugs, and observe the average mitotic rate of the surviving population. Our models predict that, if there is an initial 
increase in the average mitotic rate, followed by a decrease and a further increase, then it is likely that DTPs are present in the initial population, and stress-induced adaptation is not occurring.

\section{A4. Stem-like characteristics temporarily stabilize the subpopulation of DTPs}

Sharma and co-workers showed that the DTPs display stem-like surface markers, which are absent in the DTEPs [12]. Therefore, it is reasonable to assume that the DTPs express some stem-like characteristics. In particular, stem cells have the potential to generate more stem cells, as well as differentiated daughter cells [32] - they are capable of symmetric cell divisions, where a dividing stem cell can produce either only stem-cell daughters (selfrenewal) or only differentiated daughters, or asymmetric cell divisions, where a dividing stem cell produces one stem-cell daughter and one differentiated daughter. Under steadystate conditions, it is generally thought that stem cells divide asymmetrically. However, when a population of stem cells is subjected to chemotherapy, they have the ability to switch to a symmetric mode of division (symmetric self-renewal), in order to regenerate depleted stem-cell pools [32,33].

Motivated by these considerations, we utilize the extended I-B model to investigate the role of self-renewal and asymmetric cell divisions in the development of drug tolerance. We analyse the dynamics of the cells for a range of different values of the symmetric self-renewal probability $p_{1}$, when the probability of asymmetric self-renewal $p_{2}$ equates to $1-p_{1}$, so that the probability of symmetric differentiation $p_{3}$ is zero (see Supplementary Fig. S3).

Again we consider the two hypothetical scenarios described in Section A1. In both scenarios, if DTPs do transiently dominate the surviving population, then a higher probability of self-renewal increases the time taken for the emergence of the DTEPs from the 
DTPs. Furthermore, after the DTEPs have emerged, a higher probability of self-renewal corresponds to a larger and more stable subpopulation of DTPs in the surviving population. These predictions are illustrated in Fig. 7 where, for Scenario (i), we plot the trajectories of the mean phenotypic expression of the population and the corresponding numbers of PC9s, DTPs an DTEPs as a function of time, for three different values of $p_{1}$ (see also Supplementary Fig. S8(c)-(d) for Scenario (ii)).

On the other hand, if the DTPs do not transiently dominate the surviving population, either because the population becomes extinct before it can become drug tolerant (see Supplementary Fig. S7(c)-(d)), or because the DTEPs emerge directly from the PC9s (see Fig. S8(a)-(b)), then, not surprisingly, the probability of self-renewal $p_{1}$ does not play any role in the dynamics of the population.

\section{Discussion}

The PC9 cell line experiments reported in [12] are performed in an isolated and relatively homogeneous environment, and involve only a few constituents - a genetically identical cell population, culture media, and combinations of drugs. Furthermore, each experiment has clear observables, namely the percentage of surviving cells and their phenotypic distributions, which make the PC9 cancer cell lines an ideal system to study from a mathematical perspective.

Here, we have presented an I-B computational model and an IDE model of the evolution of phenotype observed in [12], which rely on the assumption that this evolutionary process is driven by the interplay between selection, non-genetic phenotype instability and stress-induced adaptation. Distinct mathematical strategies have been utilized to model each evolutionary mechanism. Specifically, we have modeled selection through prolifer- 
ation and death probabilities, which depend on the levels of proliferative and survival potentials of the cells, as well as on the cell micro-environment. Non-genetic phenotype instability has been modeled by stochastic fluctuations, or diffusion, in the levels of cell proliferation and survival potentials. Finally, stress-induced adaptation of cell proliferation level, which occurs in the presence of the drugs, and depends on the level of survival potential, has been modeled with an advection term. This enabled us to better understand the effect of each mechanism on the phenotypic evolution in the PC9 cell line.

The mathematical models presented here reproduce the main experimental observations detailed in [15], and also provide insight into the mechanisms controlling the dynamic variation in phenotype of the PC9 cancer cells during drug therapy, and after drug washout. Using our models as in silico laboratories, we performed mathematical analyses with two types of initial conditions, one where the population consists of only PC9s, and the other where there are also a small number of DTPs. Our analysis highlights the important role of non-genetic fluctuations in phenotype in the emergence of drug tolerance in PC9 cancer cell lines. In particular, we suggest that the absence of non-genetic instability can result in the stabilisation of the DTP phenotype in the surviving population, so that DTEPs do not emerge, or even in extinction. This is a key result since it supports the idea that epigenetic therapy may be a promising therapeutic strategy in the war against cancer [34-36]. Furthermore, it is also consistent with the experiments reported in [12], where epigenetic drugs, when used in combination with high doses of the cytotoxic therapy, were able to prevent the emergence of drug tolerance and kill the cancer cell population.

Another important prediction of our models is that the transient dominance of DTPs is strictly related to the usage of high doses of cytotoxic drugs. If experimentalists apply a lower dose of cytotoxic agents to the PC9 cell population during drug therapy, we propose 
that it would be highly unlikely to observe DTPs. Rather, we would expect the DTEPs to emerge directly from the PC9 population. Note that this is the usual way to yield stable drug-tolerant lineages [37].

Our analyses also suggest that stem-like, self-renewing cell divisions of DTPs can act to increase the number of DTPs in the system, and is consistent with other mathematical models of stem-cell dynamics [38]. In particular, we expect that DTP self-renewal can act to stabilize the DTP subpopulation for a longer period of time during drug therapy, compared to the case without self-renewal, as well as to delay the emergence of DTEPs.

Based on our models, we can conclude that if there are no DTPs present in the initial population of PC9 cells, then it is likely that a proper interplay between selection, non-genetic phenotype instability and stress-induced adaptation is mandatory for the transient appearance of the DTP phenotype during high-dose drug therapy. On the other hand, if there are some DTPs present in the initial population, and the concentration of the drugs is high enough, then selection and phenotypic fluctuations are enough to explain the experimental observations reported in [12]. Therefore, the next biologically meaningful question is: are DTPs present in the initial population? Our analysis enabled us to propose a low cytotoxic drug-dose experiment which could answer this question.

Another possible way to determine whether DTPs are present ab initio would be to utilize the experimental technique described in [39] to identify and isolate slow-cycling cells in the parental PC9 cell population, and subsequently compare the drug sensitivity of these cells with that of the more rapidly dividing PC9 cells. The presence of slowcycling, drug-tolerant cells would suggest that DTPs are present in the initial population of PC9s.

The low-dose experiment we proposed could also determine whether stress-induces the DTP phenotype. However, another way to assess whether PC9s are induced by 
stress to acquire the DTP phenotype would be to perform a fluorescence-activated cell sorting analysis on the PC9s with respect to the Cluster of Differentiation 24 (CD24) surface marker, at a time soon after drug delivery. In [12], the authors showed that the DTPs have a high level of expression of CD24, compared with the PC9s and DTEPs. Therefore, if the surviving population has an intermediate level of expression soon after drug exposure, relative to the expression of CD24 in PC9s and DTPs, then this would suggest that the DTP phenotype is a result of non-genetic fluctuations in phenotype, selection and stress-induced adaptation.

Finally, we believe that structuring cancer cell populations according to a continuous phenotype is, in general, an appropriate strategy to describe the evolutionary dynamics of cells under stress. This is particularly true for the cell population studied in [12], where phenotypes are a clear source of biological variability. In our study, we have considered a two-dimensional structuring phenotype. However, we could introduce a third phenotypic trait to represent (de-)differentiation, which may help to better characterize the evolution of the system, particularly for what concerns the dynamics following drug washout. It is then natural to wonder about what could be a biological quantitative variable underlying such a continuous (multidimensional) phenotype. We propose, as a reasonable candidate, the degree of (transient) epigenetic modifications of the DNA (methylation, histone acetylation) in relevant parts of the genome. Such modeling has been previously proposed in [40], using a one-dimensional, phenotype-like structure variable. By using a multidimensional phenotype, we propose to consider differential effects of epimutations on genes responsible for the classical intracellular pathways of proliferation, survival and (if necessary, in a three-dimensional extension of the present model) differentiation. 


\section{Acknowledgments}

\section{References}

1. Navin N, Krasnitz A, Rodgers L, Cook K, Meth J, Kendall J, et al., Inferring tumor progression from genomic heterogeneity. Genome Res (2010) 20: 68-80.

2. Gerlinger M, Rowan AJ, Horswell S, Larkin J, Endesfelder D, Gronroos E, et al., Intratumor heterogeneity and branched evolution revealed by multiregion sequencing. N Engl J Med (2012) 366: 883-892.

3. Holohan C, Van Schaeybroeck S, Longley DB, Johnston PG, Cancer drug resistance: an evolving paradigm. Nat Rev Cancer (2013) 13: 714-726.

4. Gupta PB, Fillmore CM, Jiang G, Shapira SD, Tao K, Kuperwasser C, et al., Stochastic state transitions give rise to phenotypic equilibrium in populations of cancer cells. Cell (2011) 146: 633-644.

5. Song CW, Lee H, Dings RP, Williams B, Powers J, Dos Santos T, et al., Metformin kills and radiosensitizes cancer cells and preferentially kills cancer stem cells. Sci Rep (2012) 2: 362 .

6. Yang G, Quan Y, Wang W, Fu Q, Wu J, Mei T, et al., Dynamic equilibrium between cancer stem cells and non-stem cancer cells in human sw620 and mcf-7 cancer cell populations. Brit J Cancer (2012) 106: 1512-1519.

7. Greaves M, Maley CC, Clonal evolution in cancer. Nature (2012) 481: 306-313.

8. Glasspool R, Teodoridis JM, Brown R, Epigenetics as a mechanism driving polygenic clinical drug resistance. Brit J Cancer (2006) 94: 1087-1092. 
9. Newman JR, Ghaemmaghami S, Ihmels J, Breslow DK, Noble M, DeRisi JL, et al., Single-cell proteomic analysis of s. cerevisiae reveals the architecture of biological noise. Nature (2006) 441: 840-846.

10. Brock A, Chang H, Huang S, Non-genetic heterogeneity-a mutation-independent driving force for the somatic evolution of tumours. Nat Rev Genet (2009) 10: 336-342.

11. Pisco AO, Brock A, Zhou J, Moor A, Mojtahedi M, Jackson D, et al., Non-darwinian dynamics in therapy-induced cancer drug resistance. Nat Commun (2013) 4: 2467.

12. Sharma SV, Lee DY, Li B, Quinlan MP, Takahashi F, Maheswaran S, et al., A chromatin-mediated reversible drug-tolerant state in cancer cell subpopulations. Cell (2010) 141: 69-80.

13. Aktipis CA, Boddy AM, Gatenby RA, Brown JS, Maley CC, Life history trade-offs in cancer evolution. Nat Rev Cancer (2013) 13: 883-892.

14. Silander OK, Nikolic N, Zaslaver A, Bren A, Kikoin I, Alon U, et al., A genomewide analysis of promoter-mediated phenotypic noise in escherichia coli. PLoS Genet (2012) 8: e1002443.

15. Swain PS, Elowitz MB, Siggia ED, Intrinsic and extrinsic contributions to stochasticity in gene expression. Proc Natl Acad Sci USA (2002) 99: 12795-12800.

16. Munsky B, Neuert G, van Oudenaarden A, Using gene expression noise to understand gene regulation. Science (2012) 336: 183-187. 
17. Taniguchi Y, Choi PJ, Li GW, Chen H, Babu M, Hearn J, et al., Quantifying e. coli proteome and transcriptome with single-molecule sensitivity in single cells. Science (2010) 329: 533-538.

18. Sigal A, Milo R, Cohen A, Geva-Zatorsky N, Klein Y, Liron Y, et al., Variability and memory of protein levels in human cells. Nature (2006) 444: 643-646.

19. McCullough KD, Coleman WB, Ricketts SL, Wilson JW, Smith GJ, Grisham JW, Plasticity of the neoplastic phenotype in vivo is regulated by epigenetic factors. Proc Natl Acad Sci USA (1998) 95: 15333-15338.

20. Kreso A, O’Brien CA, van Galen P, Gan OI, Notta F, Brown AM, et al., Variable clonal repopulation dynamics influence chemotherapy response in colorectal cancer. Science (2013) 339: 543-548.

21. Williams KP, Allensworth JL, Ingram SM, Smith GR, Aldrich AJ, Sexton JZ, et al., Quantitative high-throughput efficacy profiling of approved oncology drugs in inflammatory breast cancer models of acquired drug resistance and re-sensitization. Cancer Lett (2013) 337: 77-89.

22. Lackner MR, Wilson TR, Settleman J, Mechanisms of acquired resistance to targeted cancer therapies. Future Oncol (2012) 8: 999-1014.

23. Davidson JD, Ma L, Flagella M, Geeganage S, Gelbert LM, Slapak CA, An increase in the expression of ribonucleotide reductase large subunit 1 is associated with gemcitabine resistance in non-small cell lung cancer cell lines. Cancer Res (2004) 64: $3761-3766$.

24. Gautam A, Bepler G, Suppression of lung tumor formation by the regulatory subunit of ribonucleotide reductase. Cancer Res (2006) 66: 6497-6502. 
25. Anderson AR, Weaver AM, Cummings PT, Quaranta V, Tumor morphology and phenotypic evolution driven by selective pressure from the microenvironment. Cell (2006) 127: 905-915.

26. Charlebois DA, Abdennur N, Kaern M, Gene expression noise facilitates adaptation and drug resistance independently of mutation. Phys Rev Lett (2011) 107: 218101.

27. Silva AS, Gatenby RA, Research a theoretical quantitative model for evolution of cancer chemotherapy resistance. Biol Direct (2010) 5: 25.

28. Delitala M, Lorenzi T, A mathematical model for the dynamics of cancer hepatocytes under therapeutic actions. J Theor Biol (2012) 297: 88-102.

29. Lavi O, Greene JM, Levy D, Gottesman MM, The role of cell density and intratumoral heterogeneity in multidrug resistance. Cancer Res (2013) 73: 7168-7175.

30. Lorz A, Lorenzi T, Hochberg ME, Clairambault J, Perthame B, Populational adaptive evolution, chemotherapeutic resistance and multiple anti-cancer therapies. ESAIM-Math Model Num (2013) 47: 377-399.

31. Chao Y, Wu Q, Acquafondata M, Dhir R, Wells A, Partial mesenchymal to epithelial reverting transition in breast and prostate cancer metastases. Cancer Microenviron (2012) 5: 19-28.

32. Morrison SJ, Kimble J, Asymmetric and symmetric stem-cell divisions in development and cancer. Nature (2006) 441: 1068-1074.

33. Bodine DM, Seidel NE, Orlic D, Bone marrow collected 14 days after in vivo administration of granulocyte colony-stimulating factor and stem cell factor to mice 
has 10-fold more repopulating ability than untreated bone marrow. Blood (1996) 88: $89-97$.

34. Clozel T, Yang S, Elstrom RL, Tam W, Martin P, Kormaksson M, et al., Mechanism-based epigenetic chemosensitization therapy of diffuse large b-cell lymphoma. Cancer Discov (2013) 3: 1002-1019.

35. Juergens RA, Wrangle J, Vendetti FP, Murphy SC, Zhao M, Coleman B, et al., Combination epigenetic therapy has efficacy in patients with refractory advanced non-small cell lung cancer. Cancer Discov (2011) 1: 598-607.

36. Timp W, Feinberg AP, Cancer as a dysregulated epigenome allowing cellular growth advantage at the expense of the host. Nat Rev Cancer (2013) 13: 497-510.

37. Gottesman MM, Mechanisms of cancer drug resistance. Annu Rev Med (2002) 53: $615-627$.

38. Marciniak-Czochra A, Stiehl T, Ho AD, Jäger W, Wagner W, Modeling of asymmetric cell division in hematopoietic stem cells-regulation of self-renewal is essential for efficient repopulation. Stem Cells Dev (2009) 18: 377-386.

39. Moore N, Houghton J, Lyle S, Slow-cycling therapy-resistant cancer cells. Stem Cells Dev (2011) 21: 1822-1830.

40. Lei J, Levin SA, Nie Q, Mathematical model of adult stem cell regeneration with cross-talk between genetic and epigenetic regulation. Proc Natl Acad Sci USA (2014) 111: E880-E887. 


\section{Figure legends}

Figure 1. Phenotype evolution in PC9 cells during cytotoxic-drug therapy. (a) Schematic diagram of phenotype evolution in PC9 cells during high-dose cytotoxic-drug therapy. Note that, in the scenario pictured here, there are DTPs present in the initial PC9 population. However, this may not necessarily be the case. (b) Schematic diagram representing the evolution of proliferation and survival potential levels in PC9 cells during cytotoxic-drug therapy. The dashed-red lines highlight the regions of the phenotype space corresponding to the PC9s, DTPs and DTEPs.

Figure 2. Mathematical models recover the population level dynamics of the PC9

cells. (a) Trajectories of the mean phenotypic expression for $0 \leq t \leq T$. (b) Global population density as a function of time. (c) Cell distribution over the survival potential at three different times $t=T / 50, T / 10, T / 2$. (d) Cell distribution over the proliferation potential at three different times $\mathrm{t}=\mathrm{T} / 50, \mathrm{~T} / 10, \mathrm{~T} / 2$. Solid lines are the numerical results from the IDE model, while the broken lines are from the I-B model. (e)-(g) Phenotypic distribution of the cell population at three different times $t=T / 10, T / 2, T$ for the I-B model. (h)-(j) Phenotypic distribution of the cell population at three different times $t=T / 10, T / 2, T$ for the IDE model. Details are provided in the Supplementary Material.

Figure 3. Mathematical models recover the drug resensitization of (a) DTPs and (b) DTEPs following drug washout. Trajectories of the mean phenotypic expression. Here, solid lines are the numerical results from the IDE model during drug exposure, while the broken lines are from the IDE model after drug washout. Details are provided in the Supplementary Material. 
Figure 4. Non-genetic instability is crucial for the emergence of DTEPs. We illustrate the effect of the rate of phenotypic fluctuations (i.e., the parameter $\beta$ ) on (a), (c) the trajectory of the population mean trait levels for $0 \leq t \leq T$, and (b), (d) the corresponding global population density, as a function of time, in a high drug-dose regime and when there are (a), (b) only PC9s present in the initial population and stress-induced adaptation is present, or (c), (d) 98\% PC9s and 2\% DTPs present in the initial population and stress-induced adaptation is absent. Note that in panels (a) and (c), the trajectories corresponding to the case $\beta=0$, terminate (when the populations become extinct) in the regions corresponding, respectively, to the PC9 and DTP phenotype. Details are provided in the Supplementary Material.

Figure 5. A high dose of cytotoxic drugs is necessary for the transient dominance of DTPs. We illustrate the effect of the concentration of the cytotoxic drugs (i.e., the parameter $c$ ) on (a), (c) the trajectory of the population mean trait levels for $0 \leq t \leq$ $T$ (where $T$ is the end time of the simulation), and (b), (d) the corresponding global population density, as a function of time, when there are (a), (b) only PC9s present in the initial population and stress-induced adaptation is present, or (c), (d) 98\% PC9s and 2\% DTPs present in the initial population and stress-induced adaptation is absent. Details are provided in the Supplementary Material.

\section{Figure 6. Low drug-dose experiments could establish whether the DTP phenotype}

is stress-induced. Trajectories of the population mean trait levels during low drug-dose therapy, when there are $98 \%$ PC9s and 2\% DTPs present in the initial population, and 
stress-induced adaptation of the proliferation level is (a) absent or (b) present. Details are provided in the Supplementary Material.

Figure 7. Stem-like characteristics of DTPs stabilize the transiently dominant subpopulation of DTPs for longer. We illustrate the effect of the DTP self-renewal probability on (a) the trajectories of the mean phenotypic expression and (b) the corresponding number of PC9s (solid lines), DTPs (dotted lines) and DTEPs (dash-dot lines) as a function of time. Numerical results come from the extended I-B model. Details are provided in the Supplementary Material.

\section{Figures}

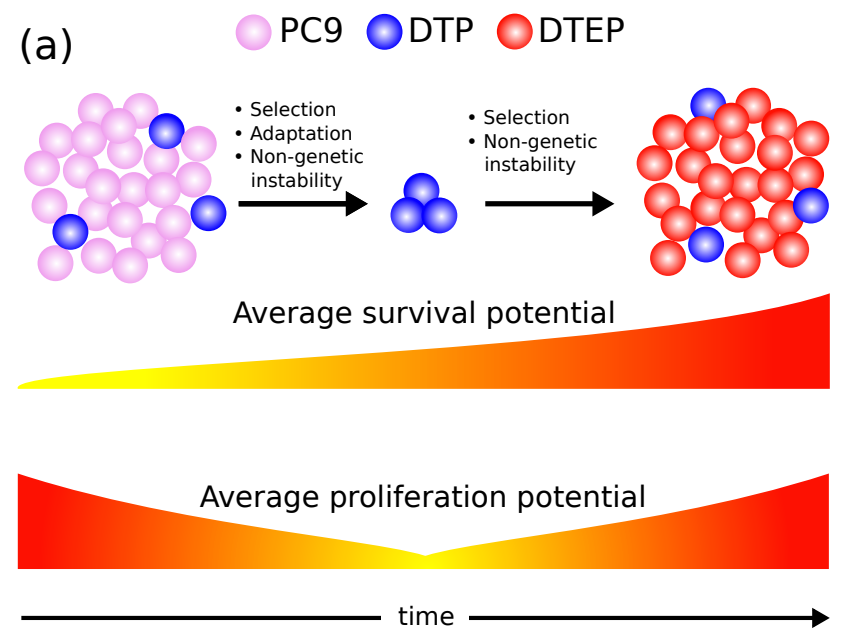

(b)

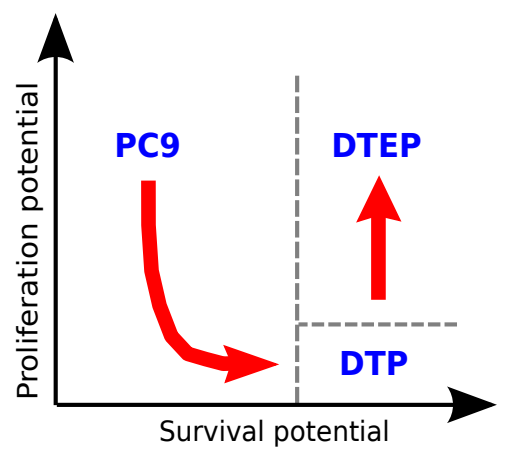

Figure 1 

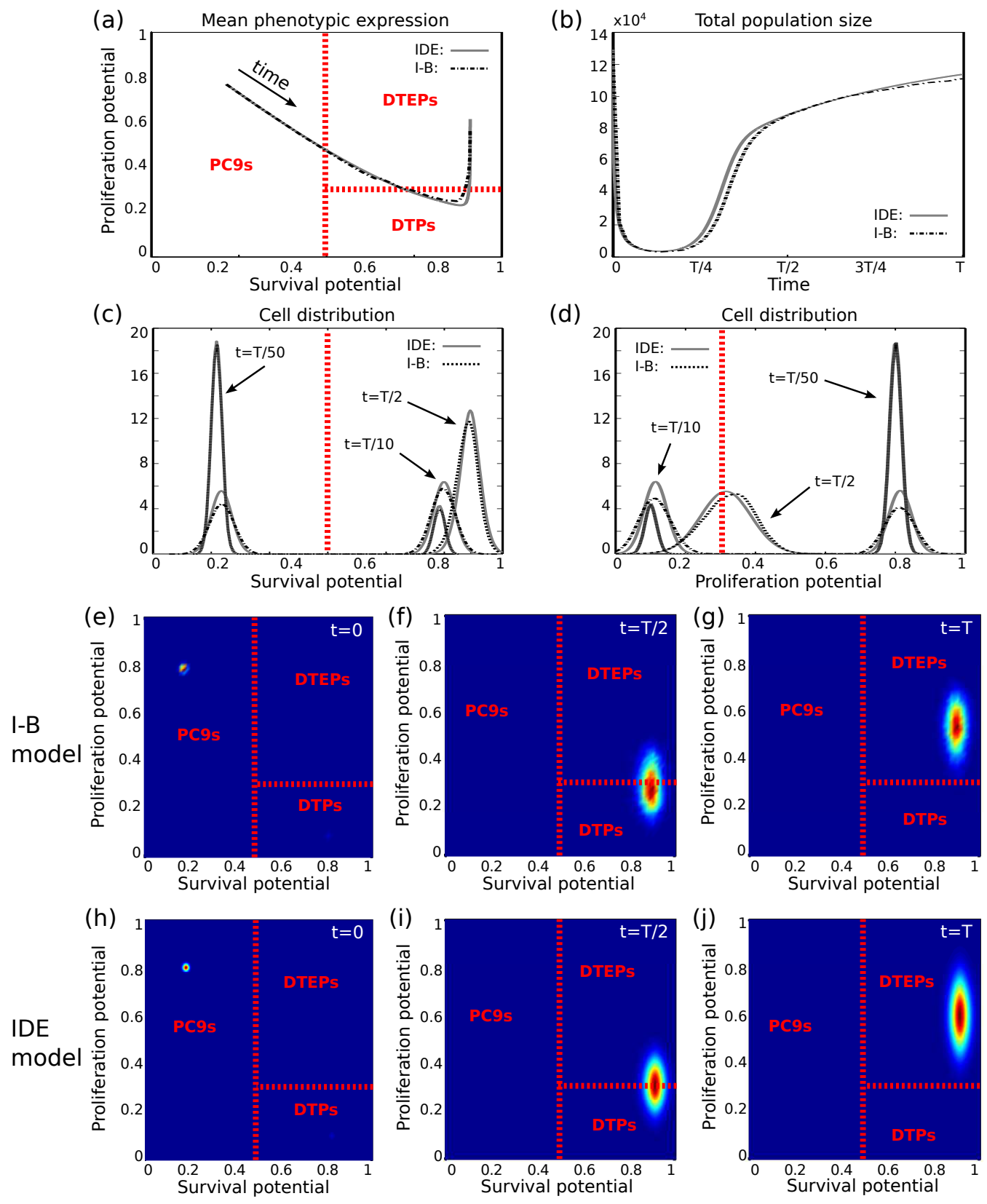

Figure 2 

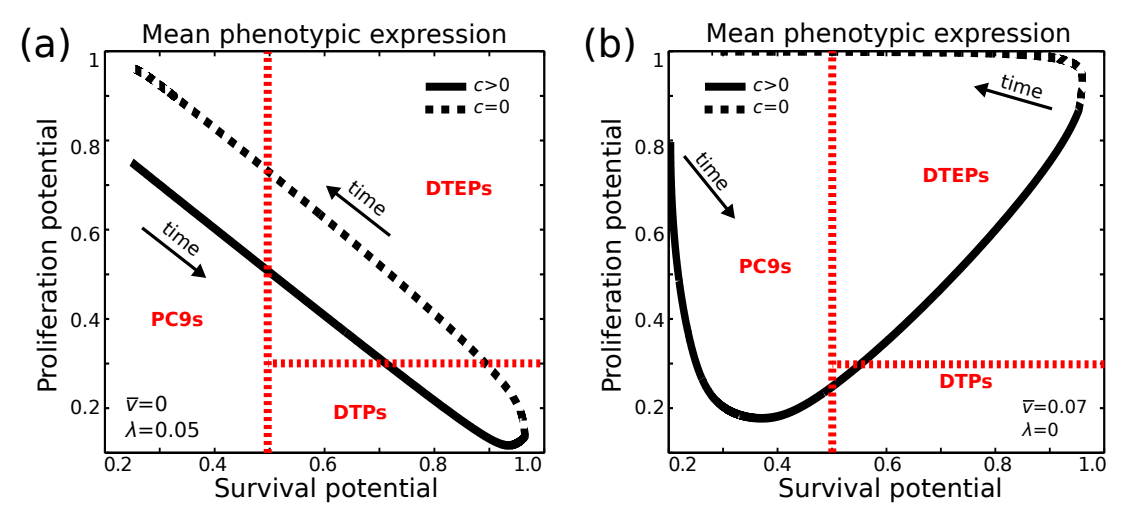

Figure 3

Only PC9s initially

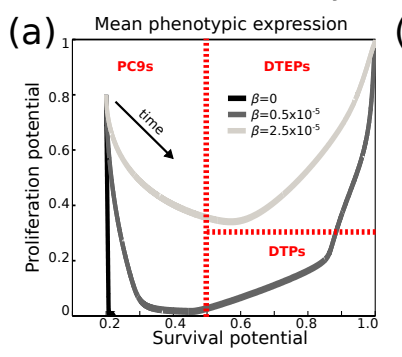

(b) ${ }_{10}^{\times 10^{4}}$ Global population density

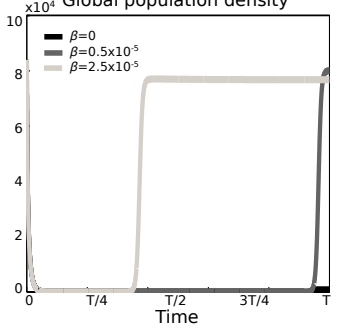

DTPs and PC9s initially

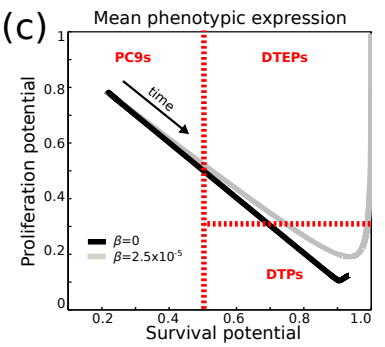

(d) ${ }_{10} \times 10^{4}$ Global population density

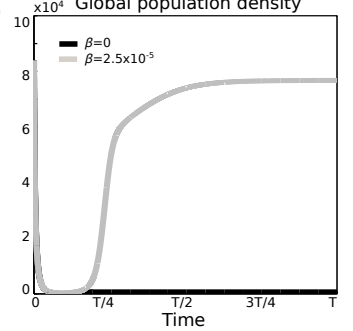

Figure 4

Only PC9s initially

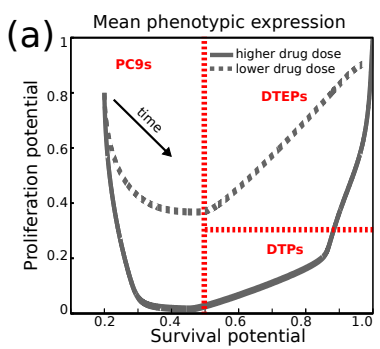

(b)

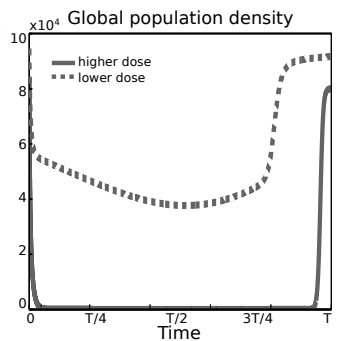

DTPs and PC9s initially
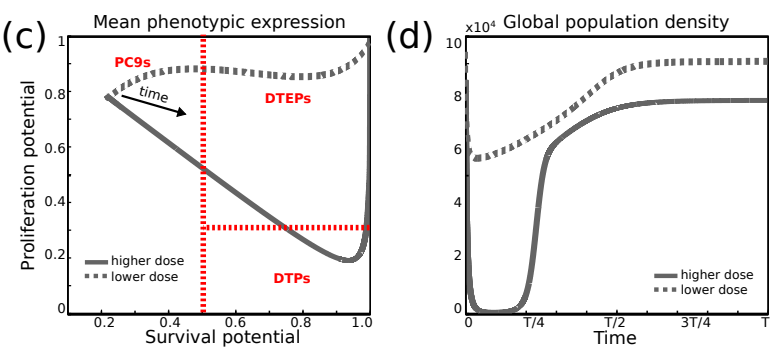

Figure 5 

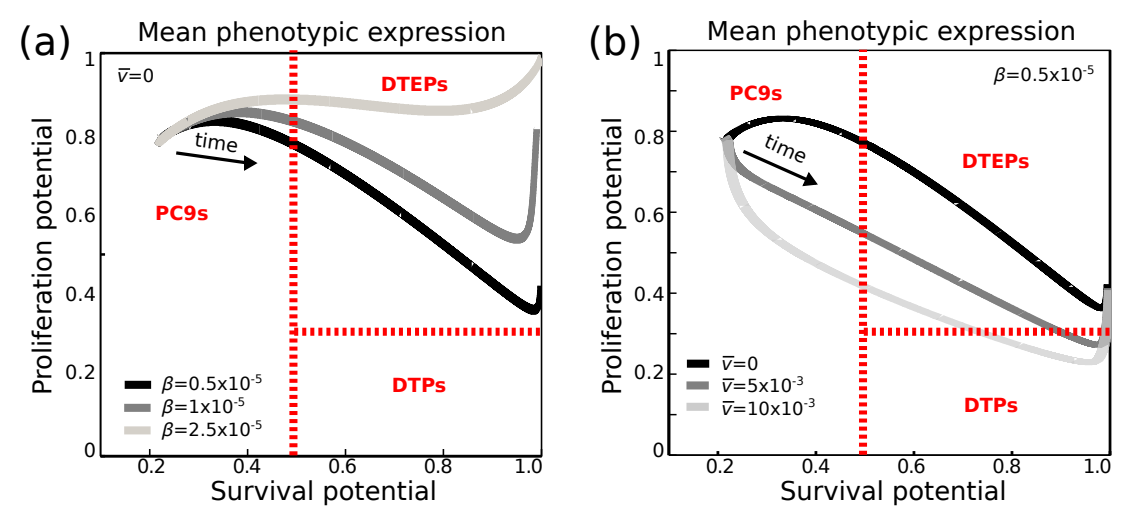

Figure 6
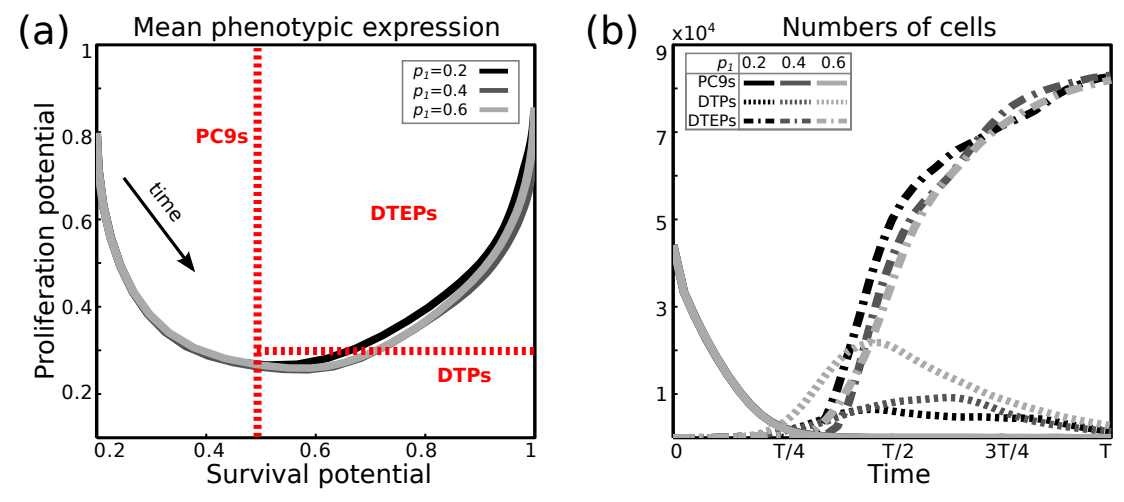

Figure 7 